\title{
LA SOSTENIBILIDAD DEL TURISMO COMUNITARIO, DESDE UNA ÓPTICA MILENARIA.
}

Christian Rivera García Mg. ${ }^{1}$, Efraín Velasteguí López. Mg. ${ }^{2}$, Dr. Yasser Vázquez Alfonso PhD. ${ }^{3}$ Lcdo. Pablo Velasteguí López. ${ }^{4}$

\begin{abstract}
The objective of this research was to assess the diagnosis and planning of tourist activities carried out in the areas of transport, accommodation, food and tourist information that is shared with domestic and foreign travelers from a millennial approach that reflects major changes in mentality Orthodox, traditional in the social hierarchy and outdated in the openness to change Community Tourism. Research plays an outstanding role in this work because community projects need a revaluation in their innovation. For this work, "research was considered "as a systematic and organized human act destined to produce knowledge, and to" community tourism planning "as the guide to think before acting, using processes to introduce greater rationalization and organization in the activities and actions of tourism, which must to Achieve their objectives and pre-established goals efficiently and effectively using resources that in some cases are scarce and in other places tested. The sustainable development approach filters parameters rescued from the activity, especially community, interested in decontextualizing the reality in their environment whose permanent participation has sown the need for rational use of the natural and cultural resources of which they have always been a part, not as owners but as an adjunct to the worldview. The timid and withdrawn sustainability has led us to the avidya of strategies without sufficiently satisfactory results; particularly from the point of view of the equitable distribution of knowledge for those who think for others and those who think for themselves disconnecting the tourist and environmental interrelation with the present and future population.
\end{abstract}

KEYWORDS: Sustainability, Tourism, Community, Community Tourism, Millennium

CÓDIGO UNESCO: Turismo 531290

\section{RESUMEN}

\footnotetext{
${ }^{1}$ Universidad Técnica de Babahoyo, Ecuador, crivera@utuutb.edu.ec

2 Ciencia Digital, Ambato, Ecuador, luisefrainvelastegui@cienciadigital.org

3 Universidad de la Habana, Cuba, yasser@ftur.uh.cu

4 Pensionado la Merced, Ambato, Ecuador, pablohomerovelastegui@ hotmail.com
} 
El objetivo de la presente investigación fue valorar el diagnóstico y la planificación, de las actividades turísticas realizadas en los ámbitos de transporte, alojamiento, alimentación e información turística que se comparte con viajeros nacionales y extranjeros desde un enfoque milenario que refleja grandes cambios de la mentalidad ortodoxa, tradicional en la jerarquización social y de la desactualización en la apertura al cambio del Turismo Comunitario. La investigación juega un papel sobresaliente en este trabajo porque los proyectos comunitarios necesitan de una revalorización en su innovación. Para este trabajo, se consideró la "investigación" como una acto humano sistemático y organizado destinado a producir conocimientos, y a la "planificación turística comunitaria" como la guía de pensar antes de actuar, utilizando procesos para introducir una mayor racionalización y organización en las actividades y acciones propias de la actividad turística, que debe alcanzar sus objetivos y metas preestablecidas de manera eficiente y eficaz utilizando los recursos que en algunos casos son escasos y en otras puestos a prueba. El enfoque de desarrollo sostenible filtra parámetros rescatados de la actividad, especialmente, comunitaria interesada en descontextualizar la realidad en su entorno cuya participación permanente a sembrado la necesidad de aprovechamiento racional de los recursos naturales y culturales de los cuales siempre han formado parte, no como dueños sino como elemento adjunto a la cosmovisión. La sostenibilidad tímida y retraída nos ha conducido al avidya de estrategias sin resultados suficientemente satisfactorios; particularmente desde el punto de vista de la distribución equitativa del conocimiento para los que piensa por otros y los que piensan por si mismos desconectando la interrelación turística y ambiental con la población presente y futura.

Palabras Claves: Sostenibilidad, Turismo, Comunitario, Turismo comunitario, Milenio

\section{INTRODUCCIÓN}

El desarrollo local se sustenta en iniciativas turísticas de sostenibilidad rural. La dinámica territorial de las comunidades indígenas desmiembran las múltiples posibilidades de formar clústeres de apoyo a iniciativas de emprendimiento que están ancladas a planificaciones previamente establecidas en diferentes ramales de organización local, regional, nacional e internacional.

Intensificar el rescate y creación de actividades recreativas y de descanso son el reto de la nueva página del Ecuador multi étnico y biodiverso. Estos esfuerzos motivan el acoplamiento de iniciativas turísticas que aglomeran ideas de jóvenes emprendedores que multiplican sus ideas buscando innovar en mercados cada vez más competitivos. 
Estos emprendimientos tienen que enfrentar a retos mucho más diferenciadores, en los cuales se contemplan los problemas como: las pérdidas humanas particularmente por el hambre y enfermedades, esto ha comprometido realizar planificaciones que brinden un desahogo de estima y control humano sobre la coraza de impunidad en sectores realmente que necesitan ayuda de transformación y apoyo a iniciativas de gran importancia.

Se tiene que recordar que el turismo no es la solución a ciertos contratiempos poblacionales, pero si es una gran ayuda para direccionar posibles soluciones de acuerdo a interés social de regeneración turística en ventaja de conservación natural y cultural con máximas oportunidades de desarrollo local.

La demanda turística responde a tendencias globalizadoras que diagnóstica, diseña, ejecuta y valora iniciativas productivas de gran calado, proporcionando reorganizar actividades para el disfrute y distracción de los viajeros.

\section{Sostenibilidad y Educación}

La Organización de las Naciones Unidas para la Educación, la Ciencia y la Cultura (UNESCO), realiza grandes campañas de viabilidad educativa frente al desarrollo sostenible, formalizando la enseñanza con miras a universalizar el desarrollo común, personalizando la ayuda internacional con políticas y programas educativos que impriman la innovación en la práctica del desarrollo sostenible.

El apoyo internacional deja de ser la manipulación obrera, direccionada a enseñar solo lo utilizable y no lo practicable frente a teorías neoliberalistas extremas. Los proyectos deben estar encaminados al apoyo sin límites del desarrollo solidario de la educación, no se obliga a enseñar se acostumbra a estudiar, es decir se permeabiliza el conocimiento, en bases de análisis, síntesis y comparaciones reales de entornos y escenarios propios del planeta, generando una clara toma de decisiones y responsabilizándose por las mismas. (Aikenhead, 1985) dirigidas al logro de un desarrollo culturalmente plural y físicamente sostenible (Delors, 1996; Cortina et al., 1998; Sachs, 2008). La educación para la Sostenibilidad persigue, en definitiva, lograr una profunda revolución de las mentalidades [...] y, solidariamente, del modelo socioeconómico vigente.

Para algunos autores, estos valores solidarios y comportamientos responsables exigen superar un "posicionamiento claramente antropocéntrico que prima lo humano respecto a lo natural" en aras de un biocentrismo que -integra a lo humano- como una especie más, en el ecosistema". (García, 1999). El antropocentrismo radicaliza al hombre sobre el aje natural y lo integra como sistema ecológico marcando el mal concebido egoísmo singular, que margina la protección del medio ambiente y su biodiversidad. 
La publicación de enceres consumistas frente a un futuro sostenible donde se requiere que prime la educación plural e integral, es la pugna actual en nuestra sociedad, la obligatoriedad de estudiar subyace al verdadero deseo de hacerlo en concordancia a la problemática unidireccional de los patrones de consumo y medios de producción arribistas. La educación requiere enfocarse en una vía transversal de salvedad para un futuro sostenible equilibrando en antropocentrismo y el biocentrismo como teorías de equilibrio global. Basta con ello para comprender que, por ejemplo, una adecuada educación ambiental para el Desarrollo Sostenible es incompatible con una publicidad agresiva que estimula un consumo poco inteligente (Mattar, 2012).

Frente a todo ello se precisa una educación que ayude a contemplar los problemas ambientales y del desarrollo en su globalidad (Tilbury, 1995; Luque, 1999; Duarte, 2006), teniendo en cuenta su estrecha vinculación y las repercusiones a corto, medio y largo plazo, tanto para una colectividad dada como para el conjunto de la humanidad y nuestro planeta (Novo, 2006a; Mattar, 2012); a comprender que no es sostenible un éxito que exija el fracaso de otros; a transformar, en definitiva, la interdependencia planetaria y la mundialización en un proyecto plural, democrático y solidario (Delors, 1996).

La responsabilidad fue dada por las generaciones que nos antecedieron y que no lo lograron, la responsabilidad es por la herencia que se entregara a las generaciones venideras, la responsabilidad está en ser consecuentes con nosotros mismos y con la defensa de nuestro hábitat del cual somos parte y no sus dueños, este el verdadero cambio cultural que necesitamos promover tanto en actitud como en comportamiento, baso conductor al cual nos lleva la educación.

La educación prevé comportamientos en contra de la vida como: el agotamiento y destrucción de los recursos, producidos por la transnacionales, industrias agobiadas por el derroche y el centralismo monetario. Pero resulta fácil mostrar (bastan cálculos muy sencillos) que si bien esos “pequeños cambios” suponen, en verdad, un ahorro energético per cápita muy pequeño, al multiplicarlo por los muchos millones de personas que en el mundo pueden realizar dicho ahorro, este llega a representar cantidades ingentes de energía, con su consiguiente reducción de la contaminación ambiental (Furió et al., 2005).

Se precisa, por tanto, un esfuerzo sistemático por incorporar la educación para la Sostenibilidad, como una prioridad central en la alfabetización básica de todas las personas, es decir, como un objetivo clave en la formación de los futuros ciudadanos y ciudadanas (Novo, 2006a), evitando planteamientos simplistas de los cambios necesarios como los que ofrecen listados de supuestas "medidas sencillas para salvar el planeta” (Maniates, 2013).

\section{Educación para la Sostenibilidad}


Ese es precisamente uno de los retos fundamentales que se nos presentan, el carácter sistémico de problemas y soluciones: la estrecha vinculación de los problemas, que se refuerzan mutuamente y han adquirido un carácter global, exige un tratamiento igualmente global de las soluciones, mediante lo que se ha denominado pensamiento sistémico (Collins, Genet y Christian, 2013).

El andamiaje de estructuras educativas no delimita el todo si no que lo agrupa para su beneficio, dando por sentado que brindar abanicos coyunturales mejora el pensamiento crítico y lo catapulta a un pensamiento benefactor con el medio ambiente y el hombre, si tratamos sosteniblemente al agua, no es el $\mathrm{H} 2 \mathrm{O}$ la que perdura en el tiempo si no sus elementos, funciones, beneficios, usos, producción, vida, es decir la educación no es un estudio simplista del agua sino una estructura vital para las generaciones.

Existen educadores que preocupados por esta manera egoísta de contrastar la educación en beneficio de los imperios y no de los vulnerables, se han concentrado en proporcionar ciertas pautas de desarrollo educativo para los países en vías de desarrollo y tercer mundistas. La educación pasa por la negligencia del perfeccionamiento profesional y el quemeimportismo estudiantil en mejorar su apreciación del mundo poniendo a la educación como un vehículo que brinda conocimientos, valores, actitudes e iniciativas desarrolladoras como habilidades investigativas claves para su comprensión.

\section{Economía y Sostenibilidad}

Existen indicadores económicos como la producción e inversión que brindan estabilidad a un grupo de empresas nacionales e internacionales, pero los indicadores ambientales también crecen en detrimento de la misma supervivencia humana. En la obra de Medaows, "Los límites del crecimiento" se cuestionan estos indicadores, que trabajan hoy por hoy por separado, masificando el crecimiento económico y minimizando el proceso biológico del planeta, que desvincula el concebido desarrollo sostenible con el desarrollo de la humanidad. Presentar propuestas orientadas a la sostenibilidad como: Desarrollo Sostenible, Economía Verde, Economía Solidaria, Economía Sostenible, Economía del Bien Común, entre otras, implica integrar la cooperación mundial, pero dejando a un lado los intereses particulares que confunden el verdadero concepto de sostenibilidad.

El incremento en los años de vida es un indicador que muestra a las claras, el cambio alimenticio que tienen los países desarrollados, una mejor dieta, la mejora en la calidad de productos agrícolas y los mejores niveles de alfabetización, pero esto contrasta con el incremento de la explotación de la madera, la pesca indiscriminada, uso ambicioso del CO2 y CO3, que acelera la destrucción de la capa de ozono y con ello los alcances ambientales desastrosos con que contamos en la actualidad llegando a un auténtico "ECOCIDIO", palabra 
con la que se hace referencia a la destrucción del medio ambiente, sus recursos y por ende del hombre.

Es más preocupante aun porque hemos sobrepasado algunos límites ( $\mathrm{CO}$, pérdida de biodiversidad, fijación de nitrógeno), que por su concatenación encadenan otros límites en secuencia a la destrucción y sobrepasar los demás, si no se adoptan urgentemente medidas correctoras. (Folke, 2013).

Por otra parte, como señala Kate Raworth (2014), "al igual que existe un límite máximo en el uso de los recursos, un 'techo ambiental' por encima del cual la degradación ambiental sería inaceptable, existe también un límite mínimo, un 'suelo social' por debajo del cual se llega a una privación humana inaceptable". Raworth enumera once necesidades sociales básicas que definirían ese suelo social y que reproducimos aquí por orden alfabético: alimentación, agua, educación, empleo, energía, igualdad de género, igualdad social (equidad), ingresos, participación, resiliencia y sanidad.

\section{Análisis de los Objetivos del Milenio.}

\section{La Pobreza}

Es marcada en la actualidad, las grandes diferencias entre países ricos y pobres, coreando al unísono por las desigualdades territoriales y sociales que pregonan los países industrializados, que asumen los países en vías de desarrollo y que sobreviven totalmente los subdesarrollados. Un hombre inestable en el aspecto alimentario, familiar, educativo, emocional, psicológico, profesional, esta propenso a rendir la mitad de sus capacidades y un poco menos.

- En las regiones en desarrollo, aproximadamente una de cada cinco personas vive con menos de 1,25 dólares al día.

- En las regiones en desarrollo, el empleo vulnerable representa el 56\% de todo el empleo, mientras que en las regiones desarrolladas es solo el $10 \%$.

- En todo el mundo, entre el 2011-2013, unos 173 millones de personas menos sufrieron de hambre crónica que en el período de 1990-1992.

- En todo el mundo, uno de cada cuatro niños menores de cinco años tiene una altura inadecuada para su edad.

- Cada día de 2013, 32.000 personas tuvieron que abandonar sus hogares para buscar protección, debido a un conflicto.

Se busca igualdad global, pero no deciden en que consiste esa igualdad si es por petróleo, por la riqueza minera, la riqueza ictiológica, la riqueza intelectual o por la riqueza compartida. Estos son algunos de los intereses ficticios que activan las normativas mundiales consolando y asistiendo a los más poderosos. 


\section{Mentalidad de Genero}

Las mujeres en la actualidad se constituyen en la brújula geopolítica del mundo, mostrando actitudes y aptitudes propias de lideresas seriamente comprometidas con el bienestar social. $\mathrm{Su}$ participación en contiendas electorales visibiliza y prioriza las funciones del matriarcado equitativo y justo, proponiendo alternativas de cambio sustancial en las políticas gubernamentales, abriendo las vías hacia gobiernos femeninos de profunda transformación, incluyendo a las mujeres en planos estelares del desarrollo de una nación y comprimiendo a otro grupo en mejorar su papel de guía intelectual y ética de la prole. Como se señala en el gráfico que a continuación se presenta:

- En 1990, en Asia meridional solo 74 niñas por cada 100 niños estaban matriculadas en la escuela primaria. En 2012, la tasa de matriculación fue la misma para las niñas que para los niños.

- En África subsahariana, Oceanía y Asia occidental las niñas todavía enfrentan barreras para ingresar a la escuela primaria y a la secundaria.

- En África septentrional las mujeres tienen menos de 1 de cada 5 trabajos remunerados en el sector no agrícola.

- Las mujeres ocupan en 46 países más del 30\% de los escaños del parlamento nacional en al menos una de las cámaras.

No cabe duda que la mujer es la artería clave de pensamiento teórico y práctico con que cuentan muchas naciones, aunque siguen siendo vilipendiadas, discriminadas, maltratadas y asesinadas por su humilde condición.

\section{Uso y Abuso de los Recursos Naturales}

El uso portentoso de los recursos naturales hace más frágil la vida en este planeta. El facilismo tecnológico de las transnacionales merma la capacidad del orden cíclico bilógico de la naturaleza.

La irresponsabilidad de países como: Estados Unidos de América y Canadá contrarios a la firma de convenios contra la emisión indiscriminada de dióxido de carbono acelera, reduce y complica notablemente la supervivencia del hombre y de las especies de plantas y animales en el planeta.

- Desde 1990, las emisiones de gases de efecto invernadero han aumentado casi un $50 \%$.

- En 2012, los ecosistemas protegidos cubrían el 14\% de las áreas terrestres y marítimas de todo el mundo. 
- Desde 1990, más de 2.300 millones de personas han logrado acceder a una fuente mejorada de agua potable, pero todavía hay 748 millones de personas que obtienen su agua de una fuente no mejorada.

- Entre 1990 y 2012, casi 2.000 millones de personas lograron acceder a instalaciones sanitarias mejoradas. Sin embargo, 1.000 millones de personas todavía defecan al aire libre.

- Un tercio de los residentes urbanos de las regiones en desarrollo vive todavía en tugurios.

Esta es la cruel realidad del ser humano frente a lo que él considera ser dueño, la naturaleza. Actualmente se han estrechado los lazos de apoyo y ayuda en todos los niveles del mundo por ejemplo la Unión Europea, la UNASUR, la CELAC, son bloques políticos y socioeconómicos regionales, con intereses conjuntos y realidades similares.

\section{TURISMO COMUNITARIO}

El Turismo Comunitario establece en la actualidad una oportunidad y una estrategia de desarrollo local para las comunidades rurales que permite aprovechar y conservar la potencialidad de los recursos naturales y culturales de manera sostenible.

Desde la década de los setenta, organismos internacionales han promovido al turismo como fórmula del desarrollo en los países de España y Portugal.

En España el turismo comunitario se centra en mejorar su situación económica y a conservar los recursos naturales de su territorio aunque no lo considera como desarrollo rural. Galacia y Cataluña son dos comunidades que tienen en común la diversidad de sus paisajes. El turismo comunitario ha implementado una diversificación en la oferta turística por las dos comunidades, mientras que la potencialidad de las comunas es diferente en preservar el paisaje, el patrimonio y la cultura.

En Portugal el turismo comunitario surge como una alternativa económica de las comunidades rurales como en Azores generan ingresos complementarios a las actividades económicas diarias, defendiendo sus costumbres y creencias, en la actualidad han sumado la capacidad de alojamiento rural, generando mayor oferta turística.

Australia y el Caribe, se destaca su importancia como fuente de riqueza de las economías nacionales, lo que ha sido fundamental para que los gobiernos adopten y promuevan el desarrollo turístico como una forma de generar empleos, inversiones y divisas.

En Australia el turismo comunitario es una alternativa diferente que beneficia a una población local principalmente a los pueblos indígenas y campesinos de zonas rurales.

Como en Victoria los aldeanos son anfitriones de los turistas en su pueblo, realizan la gestión de los servicios turísticos y comparten las ganancias. 
En el Caribe el turismo comunitario se refiere a diversificar e incrementar la economía rural, sin embargo las actividades fundamentales de los territorios como son la agricultura, la pesca, la artesanía y la gestión forestal, cobrando un importante valor puesto que en la región hay un número grande de comunidades que han creado una oferta turística propia, donde las organizaciones comunitarias deciden como realizar las actividades turísticas y como se deben distribuir lo que proviene de esta actividad.

El Turismo Comunitario en América Latina es un fenómeno que data de las dos últimas décadas y surge en un contexto de grandes cambios económicos, sociales y políticos: con un relativo incremento en zonas de excepcional belleza paisajística, dotadas de atractivos culturales y vida silvestre, ya sea en territorios comunales o en áreas públicas aledañas.

En el Salvador el turismo comunitario lo consideran como una forma de desarrollar el turismo en espacios rurales donde cooperativas, familias, pueblos indígenas y otras organizaciones comunales realizan actividades turísticas cuidando los recursos que se encuentran en su territorio, en la Cooperativa Las Lajas, en la zona del Cerro Verde, en San Ana caficultores ofrecen los servicios de la hacienda a los turistas para que conozcan la cadena de valor industrial generando ingresos para la comunidad.

En Colombia el turismo comunitario es el factor humano y cultural, se presenta como una alternativa de turismo solidario, ambiental, cultural y viable. La Ruta de Sur es un destino donde se pueden realizar actividades culturales. La zona tiene una cobertura vegetal y productiva donde la población ofrece los servicios turísticos enfocados en fomentar las costumbres, creencias y experiencias con la comunidad generando conciencia ambiental.

En Perú el turismo comunitario ofrece a los turistas la oportunidad de apreciar las experiencias y el ambiente de las comunidades, de manera planificada donde participan los pobladores, organizando las actividades turísticas con el objetivo de ofrecerle beneficios a la comunidad mostrando a los turistas lo que realizan en su localidad. En la comunidad de Cuyuni ofrece al turista aprendizajes interculturales, ceremonias andinas, caminatas y actividades agrícolas donde el visitante puede participar en el diario vivir de la comunidad.

Esta actividad se va posicionando como un nuevo elemento en la oferta turística, se lo considera, como uno de los múltiples productos turísticos que se ofrecen en el mercado. Las comunidades en el área rural ofrecen: vacaciones con relación naturaleza y cultura, observación de flora y fauna, convivencia con la población, aprendizaje de sus tradiciones, actividades deportivas como el senderismo, que se realizan en la mayoría de países donde se oferta esta clase de turismo.

La historia del país inicia con la aparición de los Incas que a finales del siglo XV llegaron al territorio ecuatoriano, la presencia inca fue de apenas 80 años al norte de los Andes, la conquista Inca de los pueblos no solo se dio mediante las guerras sino también por alianzas 
comerciales y matrimoniales. En la llegada de los españoles, el Tahuantinsuyo se encontraban en conflictos internos lo que facilito la conquista de los españoles.

En el Ecuador, al turismo comunitario es una alternativa, porque se lo considera un país con gran biodiversidad, donde se encuentra las cadenas montañosas como la cordillera de los andes que ocupa la zona occidental de América de Sur, la cordillera Real que es la más grande de ellas por la cadena volcánica que se comunica con la cordillera central al sur de Perú y con la cordillera central al norte de Colombia y la cordillera occidental se extiende por todo el país de norte a sur.

Las corrientes marinas en el Ecuador varían según sus movimientos la corriente Sur ecuatorial que se mueve hacia el oeste a ambos lados del Ecuador y su límite es la contra corriente ecuatorial, la corriente del Niño, se caracteriza por ser de aguas cálidas provocando el aumento de la temperatura superficial del mar, la corriente de Humboldt es compleja por tener distintos componentes: la corriente costera que se mueve cerca de la costa es la corriente oceánica más lejana y la contracorriente del Perú, en la corriente Oceánica de Humboldt tiene una afluencia más profunda y transporta aguas frías de baja salinidad. La contra corriente del Perú se caracteriza por que se desplaza en sentido opuesto al norte entre las ramas costeras y oceánicas de Humboldt es más cálida y salina. La corriente costera de Humboldt es la que marca el inicio del cambio de estación en el país disminuyendo la temperatura superficial del mar y del aire. La Sub corriente ecuatorial se caracteriza por su flujo que llega a las Islas Galápagos dirigiéndose hacia el sureste, alimentando los afloramientos a lo largo de la costa al norte de Perú. Los vientos Alisios son los que soplan los trópicos hacia el Ecuador como también los vientos Continentales son periódicos y estacionales, vierten su dirección con el paso de los días y noches con la sucesión de las estaciones.

En el Ecuador conviven sociedades, culturas, grupos étnicos, pueblos y distintas nacionalidades, con lenguas, tradiciones históricas, cosmovisiones, formas de organización socio-políticas e identidades propias y diferenciadas, que hacen de este, un país multicolor, diverso, de múltiples rostros, donde se destaca por la solidaridad y generosidad de su gente, su identidad es el sello de la personalidad que los ecuatorianos tenemos por ser nuestra historia de vida que se relaciona con la época y el lugar en donde vivimos siendo fieles a nuestras creencias y costumbres que son arraigadas desde nuestros aborígenes que vinculan con la naturaleza y las leyes del universo.

Las Mincas que se realizan en el país son para generar conciencia a los grupos de comuneros para incursionar en el turismo comunitario, las que se realizan en la región Andina se caracterizan por crear lazos fraternales con los otros pueblos y comunidades en la región Litoral se enfocan en tener beneficios propios, pero en la región Amazónica las Mincas sirven para fortalecer las costumbres ancestrales de las comunidades siendo amables entre ellos. 
Al realizar la actividad comunitaria despliega al turismo con una percepción diferente con una ideología que de una manera u otra colisionan con el sector empresarial privado, en las comunidades el turismo no suple a las principales fuentes de trabajo que son la agricultura y la ganadería en las actividades que se han dedicado durante toda su vida siendo esta una alternativa para incrementar sus ingresos reduciendo la desigualdades, siendo culturalmente enriquecedor y ambientalmente amable, al acceder que las comunidades rescaten y den a conocer su cultura, costumbre, tradiciones, historia, naturaleza y belleza escénica compartiéndola con el mundo de tal manera que la comunicación que se establezca con la comunidad y el turista, motiva al deseo de conocer su cultura y la necesidad de conservarla.

Hay turismo comunitario en áreas protegidas de las 54 el $60 \%$ hacen turismo comunitario, el $45 \%$ hacen actividades comunitarias donde no han dado pasos fundamentales, pero el objetivo es conocer las culturas de las comunidades locales enfocado en ellos y en la historia cultural.

En el litoral el ejercicio de actividades turísticas comunitarias se ha organizado y han formado comités que les permite defender sus derechos y proteger el territorio, realizando trabajos direccionados a la religión y educación de la comunidad, construyendo casas comunales, museos arqueológicos, museos in situ administrados por la comunidad siendo la visita de los turistas el factor importante para dar a conocer su patrimonio, fomentando la oferta turística por medio del turismo comunitario sin explotar inconscientemente los recursos naturales.

Sus características son: el clima cálido sub húmedo con una temperatura de $28^{\circ} \mathrm{C}$, posee gran riqueza en organismos y especies endémicas, la fauna de esta región se compone de mamíferos menores y la flora tiene componentes arbóreos de baja estatura.

En los Andes el turismo comunitario brinda el mejoramiento de la calidad de vida a familias indígenas que compartiendo sus viviendas con los turistas, permiten conocer afondo sus costumbre, creencias, conocimientos y su forma de vida, esta forma de turismo proporciona al turista que experimente como se vive dentro de las comunidades indígenas conociendo sus actividades y sus costumbres de culturas centenarias, protegiendo el manejo adecuado de los recursos naturales y territoriales de las nacionalidades y pueblos.

Son los propios pobladores los que se convierten en guías y las ganancias del turismo se establecen según las necesidades y prioridades de las comunidades resueltas en asambleas comunitarias, como por ejemplo para proyectos sociales, de salud, medio ambiente y cultura.

Sus características son impresionantes por sus zonas calientes, templadas y frías con valles de diferentes altitudes y climas, las elevaciones volcánicas y nevados hacen que esta región sea rica en flora, fauna e historia. Las ciudades de Quito y Cuenca son de gran importancia histórica como los centros artesanales en Otavalo. 
En la amazonia el nacimiento del turismo comunitario, que en un primer momento es una actividad que pueden realizar todas las comunidades para proteger y recuperar sus recursos naturales, rescatar su legado cultural y generar ingresos económicos.

Los diferentes grupos étnicos como; achuar, cofanes, quichuas, tagaeri, andoas, huaorani, shuar, sionas, zapara, secoya, shiwiar, quichua amazónico: quichua del Napo y quichua de Pastaza, son etnias, que aún conservan su estilo de vida, su belleza y sus tesoros naturales son un testimonio vivo para la salud de la selva.

El turismo comunitario se ha organizado en grupo de nativos, que con la diversidad de la flora y fauna muestran la magia que tienen la selva sus costumbres y tradiciones a los turistas, tienen centros de interpretación donde se conoce sus bailes tradicionales y como aprender a cocinar ancestralmente, los alimentos los proveen de sus cultivos, los empleados son los miembros de la comunidad y el capital que se genera es invertido tanto en el hotel como en proyectos de educación, salud y vivienda.

Las características de esta región es que la temperatura está dentro de los 15 y $40^{\circ} \mathrm{C}$ teniendo un bosque tropical lluvioso con hábitat vegetal y animal muy rico, su diversidad de etnias dictan su sentido de supervivencia.

En la región Insular no se puede realizar turismo comunitario por la capacidad de carga de los sitios visitados en el PNG, al realizar esta actividad generaría un impacto negativo sobre los sitios por la erosión, la perturbación de la fauna y la destrucción vegetal generando efectos drástico a largo plazo tanto a la población como a los animales.

\section{CONCLUSIONES}

- La sostenibilidad no es un argumento a posteriori, es la salvedad de circunstancias integradas e imbricadas de calidad de vida de la población actual para resguardar la permanencia y el modo de vida de las generaciones futuras en armonía con la naturaleza.

- El Turismo Comunitario se fundamenta en las vivencias realizadas a diario en poblaciones rurales que fortalecen la identidad cultural, mediante el respeto de sus costumbres, tradiciones y saberes ancestrales, posibilitando el intercambio de experiencias vivenciales e interculturales.

- Existen nuevos bienes, productos, servicios y procesos turísticos comunitarios que etiquetan este tipo de turismo desde innovaciones emprendedoras, fortaleciendo la actividad comunitaria e inclinando las acciones de conservación de los recursos de flora y fauna en beneficio de la población rural que maneja sus marcas culturales con apremio turístico hacia el logro de objetivos globales dentro de un ambiente de desarrollo local. 
RECEIVED: September, 2017

REVISED: December, 2017

\section{REFERENCIAS BIBLIOGRÁFICAS}

- Abrahamsson, A. (2006). "Sustainopreneurship-business with a cause". En Frostell, B. (Ed.), Science for sustainable development starting points and critical reflections. Swedish Society for Sustainable Development, VHU - Uppsala, pp. 21-30.

- Abrahamsson, A. (2008). "Sustainopreneurship -business with a cause: the promise of creative business organizing for sustainability". En Johannisson \& Lindholm Dahlstrand (Eds.), Bridging the functional and territorial views on regional entrepreneurship and development. Swedish Foundation for Small Business Research, Sweden, pp. 137-155.

- AECI - Agencia Española de Cooperación Internacional. (2006). "Manual de turismo sostenible Araucaria XXI. Fundamentos y buenas prácticas". Ministerio de Asuntos Exteriores y Cooperación, Madrid.

- Bramwell, B. \& Sharman, A. (1999). "Collaboration in local tourism policymaking". Annals of Tourism Research, 26 (2): 392-415.

- Carree, M. A. \& Thurik, R. (2003). "The impact of entrepreneurship on economic growth". En: Audretsch, D. B. \& Acs, Z. J. (Eds.), Handbook of Entrepreneurship Research. Kluwer Academic Publishers, Boston/Dordrecht, pp. 437-471.

- Cohen, B. \& Winn, M. I. (2007). "Market imperfections, opportunity and sustainable entrepreneurship". Journal of Business Venturing, 22 (1): 29-49.

- Consejería de Turismo, Comercio y Deporte. (2007). "Registro de Turismo de Andalucía", Junta de Andalucía, Sevilla.

- Dean, T. J. \& McMullen, J. S. (2007). "Toward a theory of sustainable entrepreneurship: reducing environmental degradation through entrepreneurial action". Journal of Business Venturing, 22 (1): 50-76

- Eagles, P. F.; McCooland, S. \& Haynes, C. (2002). "Sustainable tourism in protected areas: Guidelines for planning and management". UICN Gland \& Cambridge

- Epler, M. (2002). "Ecotourism: principles, practices \& policies for sustainability". United Nations Environment Programme (UNEP), Paris

- EUROPARC-España. (2009). "Anuario EUROPARC-España del estado de los espacios naturales protegidos 2009". Ed. Fundación Fernando González Bernáldez, Madrid

- EUROPARC Federation. (1999). "La Carta Europea del Turismo Sostenible en los espacios protegidos" texto oficial, Generalitat de Catalunya, Departament de Medi Ambient, Barcelona 
- EUROPARC Federation. (2007). "European Charter for Sustainable Tourism in Protected Areas", Internet: www.european-charter.org. Acceso: 11 de febrero 2011.

- Fernández, A. \& Santos, E. L. (2010). "Turismo y parque naturales en Andalucía tras veinte años desde su declaración. Análisis estadístico, tipología de parques y problemática de la situación actual". Anales de Geografía 30 (1): 29-54.

- Gerlach, A. (2003) "Sustainable entrepreneurship and innovation". Centre for Sustainability Management, University of Lueneburg, Conference Proceeding of Conference Corporate Social Responsibility and Environmental Management, Leeds.

- Hart, S. L. (2005). "Capitalism at the crossroads: the unlimited business opportunities in solving the world's most difficult problems". Wharton School Publishing, Philadelphia.

- Hassan, S. S. (2000). "Determinants of market competitiveness in an environmentally sustainable tourism industry". Journal of Travel Research, 38 (3): 239-245.

- Hockerts, K. (2003). "Sustainability innovation: ecological and social entrepreneurship and the managing of antagonistic assets". $\mathrm{PhD}$ Dissertation, University of St. Gallen, Schweiz.

- IET - Instituto de Estudios Turísticos. (2009). "Movimientos turísticos en fronteras" (Frontur) y "Movimientos turísticos de los españoles" (Familitur). Ministerio de Industria, Turismo y Comercio, Madrid.

- Ley 4/1989 de Conservación de los Espacios Naturales y de la Flora y Fauna Silvestre. BOJA No. 74, 28/3/1989.

- Nepal, S. K. (2000). "Tourism in protected areas". Annals of Tourism Research, 27 (3): 661-681.

- OMT- Organización Mundial de Turismo. (1980). "Declaración de Manila sobre el Turismo Mundial", Manila

- OMT. (1989). "Declaración de la Haya sobre turismo". La Haya.

- OMT. (1993). "Tourism the year 2000 and beyond qualitative aspects", Paris.

- OMT. (1995). "Carta Mundial de Turismo Sostenible", Conferencia Mundial de Turismo Sostenible, Lanzarote.

- Pastakia, A. (1998). "Grassroots ecopreneurs: change agents for a sustainable society". Journal of Organizational Change Management, 11 (2): 157-173.

- Poggi, M.; Ferreira, M. \& Ruschmann, D. (2006). "Competitividad y turismo sustentable. El caso de Aguas de San Pedro (San Pablo, Brasil)". Estudios y Perspectivas en Turismo, 15: 220-235.

- Pulido, J. I. (2008). "Gestión turística activa y desarrollo económico en los parques naturales andaluces. Una propuesta de revisión desde el análisis del posicionamiento de sus actuales gestores". Revista de Estudios Regionales, 81: 171-203.

- Prahalad, C. K. (2004). "The fortune at the bottom of the pyramid-eradicating poverty through profits". Wharton School Publishing, Philadelphia. 
- Schaper, M. (Ed.) (2005). "Making ecopreneurs: developing sustainable entrepreneurship". MPG Books, Bodmin, Cornwall

- Secretaría General de Turismo. (2004). "El turismo de naturaleza en España y plan de impulso. Estudios de Productos Turísticos", Ministerio de Industria y Comercio, Madrid.

- Shepherd, D. A. \& Patzelt, H. (2010). "The new field of sustainable entrepreneurship: studying entrepreneurial action linking «What is to be sustained» with «What is to be developed»". Entrepreneurship, Theory and Practice, 35 (1): 137-163.

- Steyaert, C. \& Hjorth, D. (2006). "Entrepreneurship as social change. A third movement in entrepreneurship book". Edward Elgar, Cheltenham.

- Toledano, N. \& Gessa, A. (2002). "El turismo rural en la provincia de Huelva. Un análisis de las nuevas iniciativas creadas al amparo de los programas LEADER II Y PRODER". Revista de Desarrollo Rural y Cooperativismo Agrario, 6: 107-121.

- Zahra, S. A.; Filatotchev, I. \& Wrigth, M. (2009). "How do threshold firms sustain corporate entrepreneurship? The role of boards and absorptive capacity". Journal of Business Venturing. 24 (3): 248-260 Links ] Helvetica, sans-serif"

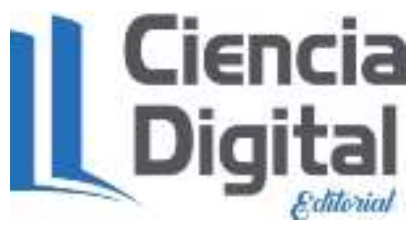




\section{PARA CITAR EL ARTÍCULO INDEXADO.}

Rivera C., Velasteguí E., Vázquez Y. \&Velasteguí P. (2018). La sostenibilidad del turismo comunitario, desde una óptica milenaria. Revista electrónica Ciencia Digital 2(1), 390-405. Recuperado desde: http://www.cienciadigital.org/index.php/CIENCIADIGITAL/article/view/102/85

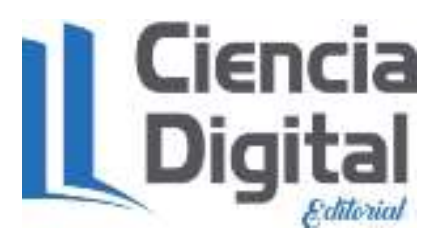

El artículo que se publica es de exclusiva responsabilidad de los autores y no necesariamente reflejan el pensamiento de la Revista Ciencia Digital.

El articulo queda en propiedad de la revista y, por tanto, su publicación parcial y/o total en otro medio tiene que ser autorizado por el director de la Revista Ciencia Digital.
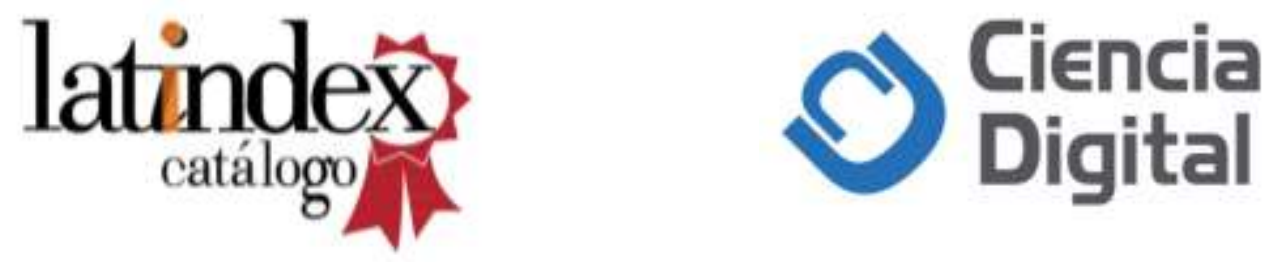